\title{
Ribozyme-mediated inhibition of caspase-12 activity reduces apoptosis induced by endoplasmic reticulum stress in primary mouse hepatocytes
}

\author{
SHAN JIANG ${ }^{1}$, QING XIE ${ }^{1}$, HUIJUAN ZHOU ${ }^{1}$, WEI ZHANG ${ }^{2}$, \\ XIAQIU ZHOU ${ }^{1}$, GUANGMING $\mathrm{LI}^{3}$, YI SHI ${ }^{4}$ and YOUXIN JIN ${ }^{4}$ \\ ${ }^{1}$ Department of Infectious Disease, Ruijin Hospital, Shanghai Jiao Tong University School of Medicine, Shanghai 200025; \\ ${ }^{2}$ Department of Infectious Disease, No. 6 People's Hospital, Shanghai Jiao Tong University, Shanghai 200233; \\ ${ }^{3}$ Department of Gastroenterology, Xinhua Hospital, Shanghai Jiao Tong University School of Medicine, Shanghai 200092; \\ ${ }^{4}$ State Key Laboratory of Molecular Biology, Institute of Biochemistry and Cell Biology, Shanghai Institutes for \\ Biological Sciences, Chinese Academy of Sciences, Shanghai 200031, P.R. China
}

Received July 22, 2008; Accepted September 12, 2008

DOI: 10.3892/ijmm_00000077

\begin{abstract}
Apoptosis via endoplasmic reticulum (ER) stress has been reported in many cell lines. ER stress plays an important role in many liver diseases and caspase-12 is the central player in ER stress-induced apoptosis. We conducted an investigation to determine whether catalytic cleavage of caspase-12 mRNA by hammerhead ribozymes can protect liver cells from apoptosis induced by ER stress. Thapsigargin (TG) was used to induce primary mouse hepatocytes apoptosis to establish the experimental system of ER stressmediated apoptosis. The effective ribozyme-Rz138 selected in vitro was embedded in eukaryotic expression vector and transfected into cultured cells. The activity of Rz138 in primary mouse hepatocytes was determined by testing the expression of caspase-12 mRNA and procaspase-12 protein in ribozyme treated cells compared with the control. The anti-apoptotic effect was assayed by the nuclear morphological features of primary mouse hepatocytes stained with Hoechst 33258 under the fluorescence microscope. Primary mouse hepatocytes were incubated with $4 \mu \mathrm{mol} / 1 \mathrm{TG}$, the percentage of apoptotic cells increased along with the treatment
\end{abstract}

Correspondence to: Professor Qing Xie, Department of Infectious Disease, Ruijin Hospital, Shanghai Jiaotong University School of Medicine, No. 197 Ruijin Er Road, Shanghai 200025, P.R. China

E-mail: xieqingrj@yahoo.com.cn

Professor Youxin Jin, State Key Laboratory of Molecular Biology, Institute of Biochemistry and Cell Biology, Shanghai Institutes for biological Sciences, Chinese Academy of Sciences, 320 Yue Yang Road, Shanghai 200031, P.R. China

E-mail: yxjin@sibs.ac.cn

Key words: apoptosis; endoplasmic reticulum stress; caspase-12; ribozyme time, obvious apoptosis was observed after $4 \mu \mathrm{mol} / 1 \mathrm{TG}$ treatment for $30 \mathrm{~h}$. Expression of caspase-12 mRNA and procaspase-12 protein were decreased significantly in hepatocytes transfected with pRz138 compared with those untransfected. The percentage of apoptotic cells was also decreased in pRz138 treated cells measured by staining with Hoechst 33258. Rz138, as a specific inhibitor of caspase-12 can down-regulate the expression of caspase-12 in primary mouse hepatocytes and protect the cells from apoptosis induced by TG. These results further elucidated the new treatment for diseases associated with ER stress-mediated apoptosis.

\section{Introduction}

Apoptosis was originally used to describe a unique type of cell death that exhibits a distinct set of morphological and biological changes, including cytoplasmic membrane blebbing, nuclear condensation, and fragmentation of chromosomal DNA. It is a crucial biological process for the development and homeostasis of all multicellular organisms (1). Activation of death receptors and mitochondrial damage are welldescribed common apoptosis pathways. Apoptosis via endoplasmic reticulum (ER) stress has been reported in many cell types (2-6). ER stress plays an important role in many liver diseases including non-alcoholic steatohepatitis, cholestasis and alcohol-induced liver disease. The pathogenesis of chronic HBV and HCV infection may also be associated with ER stress (7-13).

ER is a principal site for protein synthesis and folding and also serves as a cellular storage site for calcium. Perturbation of $\mathrm{Ca}^{2+}$ homeostasis, increased production of free radicals, inhibition of protein glycosylation, and accumulation of misfolded proteins in the ER can all elicit cellular stress responses, particularly ER stress signals. A variety of agents, including calcium ionophore, inhibitors of glycosylation, toxin, and oxidative stress can induce ER stress and lead to cell death $(14,15)$. Thapsigargin (TG) is a $\mathrm{Ca}^{2+}$ adenosine 
triphosphatase inhibitor and disrupts intracellular calcium homeostasis. TG has been used to establish experimental systems of ER stress mediated apoptosis in many studies (1619).

The caspase family of proteases is considered the central player in all apoptotic events in mammals. As with many other cellular proteases, all caspases are first synthesized as inactive pro-enzymes that can be activated upon apoptotic stimulation. It is believed that apoptosis was resulted from the proteolysis of various cellular components initiated by activated caspases $(20,21)$. Studies have revealed that caspase-12 is the central player in ER stress-mediated apoptosis. Caspase-12-/- cells were more resistant to ER stress-induced apoptosis. It has been demonstrated in many cell lines that selective down-regulation of caspase- 3 by ribozyme can protect cells against apoptosis and caspase- 12 was the upper activator of caspase-3 in ER stress-mediated apoptosis (2,22-25). Catalytic cleavage of caspase-12 mRNA may protect liver cells from apoptosis induced by ER stress.

Hammerhead ribozyme can sequence-specifically cleave target mRNA at a nucleotide phosphodiester bond resulting in two fragments, one bearing a $5^{\prime}-\mathrm{OH}$, and the other a nucleoside 2', 3'-cyclic phosphate. Divalent metal ions are essential for optimal catalytic activity, and as a general rule, substrate cleavage specificity is defined by an NUX triplet, where $\mathrm{N}$ can be any nucleotide and $\mathrm{X}$ can be $\mathrm{A}, \mathrm{U}$ or $\mathrm{C}$, but not $G$ (26). It is simple in structure with high turnover in cleavage reaction and has been widely used to inhibit endogenous gene expression of basic biochemical pathways such as angiogenesis and apoptosis. It is the first ribozyme to be approved for use in clinical trial $(27,28)$.

In the present study, TG-induced ER stress model of apoptosis in primary mouse hepatocytes was established. Rz138 was selected as an effective caspase-12 inhibitor in our previous study through in vitro cleavage reaction (29). Rz138 was embedded in eukaryotic expression vector and transfected into primary mouse hepatocytes to testify its activity on caspase-12 expression and effect on ER stressmediated apoptosis.

\section{Materials and methods}

Isolation and culture of primary mouse hepatocytes. Primary hepatocytes were isolated from 6 to 8 week-old male Balb/c mice (purchased from the Center of Animal Laboratory, SIBS, CAS) by the 2-step collagenase hepatic portal perfusion technique established by Klaunig (30) with modification. In brief, after the mouse was anaesthetized with diethyl ether, a midline laparotomy was performed. The portal vein was cannulated and perfused at a flow rate of $15 \mathrm{ml} / \mathrm{min}$, first with calcium and magnesium free Hanks balanced salt solution (HBSS) maintained at $37^{\circ} \mathrm{C}$ containing $0.5 \mathrm{mmol}$ ethylene glycol-bis-(b-aminoethyl) N, N9-tetraacetic acid (EGTA) and 0.05 mol N-2-hydroxyethylpiperazine-N-2-ethane sulfonic acid (HEPES, pH 7.35) for 3 min. Then perfused with HBSS containing $0.05 \%$ collagenase for hepatocytes isolation. The Hanks' and collagenase solutions were allowed to run as waste through a cut made in the subhepatic inferior vena cava. Hepatocytes were removed by mechanical dissociation with fine forceps in ice cooled medium, filtered through sterile $100 \mu \mathrm{m}$ stainless steel mesh to remove tissue debris, cell suspension was purified by differential centrifugation using $45 \%$ percoll (500 rpm $10 \mathrm{~min}$ ) and washed three times by centrifugation at $500 \mathrm{rpm}$ for $1 \mathrm{~min}$ at $4^{\circ} \mathrm{C}$ in PBS. Hepatocytes viability consistently exceeded $97 \%$ shown by $0.3 \%$ trypan blue exclusion.

Cells were plated at a density of $1.5-3 \times 10^{5}$ cells $/ \mathrm{ml}$ in 6well plates and cultured in Dulbecco Modified Eagle's Medium (DMEM)(Life Technology, USA) containing 10\% v/v fetal bovine serum (Invitrogen), $3 \mathrm{mmol} \mathrm{L-glutamine} \mathrm{(Life}$ Technology), HGF $10 \mathrm{ng} / \mathrm{ml}$, dexamethasone $10 \mu \mathrm{mol} / \mathrm{l}$, insulin $5 \mu \mathrm{g} / \mathrm{ml}, 50 \mathrm{U} / \mathrm{ml}$ penicillin and $50 \mu \mathrm{g} / \mathrm{ml}$ streptomycin in a humidified $5 \% \mathrm{CO}_{2}$ incubator at $37^{\circ} \mathrm{C}$. The medium was changed the following day and every 2 days thereafter.

Plasmids construction. The hammerhead ribozymes were designed according to the computer software compiled by Professor Chen Nong-An (Shanghai Institute of Biochemistry of the Chinese Academy of Science). The specificity of ribozymes for caspase-12 was determined by Blastn analysis of all the mouse gene sequences currently in GeneBank of NCBI. Thus, the ribozyme is specific for caspase-12 alone theoretically, and has no effect on other mouse genes. The gene sequences of Rz138 are shown in Table I. It was reported that G5 to A alteration in the catalytic core of hammerhead ribozyme would generate inactive ribozyme (31-33). As many studies have demonstrated that inactive mutant ribozymes have no cleavage activity (34-36), we preferred to use ribozyme without mutant in the present study.

The synthesized oligonucleotides encoding ribozymes were annealed after heating to $95^{\circ} \mathrm{C}$ for $5 \mathrm{~min}$ followed by slow cooling to room temperature. The double-stranded DNA with 5' XbaI and 3' BamHI cohesive ends, was cloned into the equivalent restriction sites of neorpBSKU6 plasmid and embedded in U6 SnRNA context containing U6 SnRNA promoter/enhancer and terminator. For further measurement of transfection efficiency, the selected ribozyme in vitro with the U6 sequence alongside was reconstructed into pEGFP-C1 vector (Clontech) with reporter gene, the newly constructed plasmid confirmed by sequencing was named pRz138 (29).

Detection of apoptosis in primary mouse hepatocytes induced by $T G$. After 4 days of isolation the culture medium was replaced with DMEM supplemented with $2 \mu \mathrm{mol} / 1$ or $4 \mu \mathrm{mol} / 1$ TG for $18,24,30$ and $48 \mathrm{~h}$ to induce ER stress-mediated apoptosis.

The cells were examined daily for morphological changes using a phase-contrast microscope. DNA was extracted using an easy DNA kit (Invitrogen). Purified DNA was resolved by electrophoresis on $1.6 \%$ agarose gels containing $0.5 \mu \mathrm{g} / \mathrm{ml}$ ethidium bromide, and visualized by ultraviolet illumination to detect DNA fragmentation.

Cells started on treatment with TG at different time points were harvested by trypsinization for $1 \mathrm{~min}$ at $37^{\circ} \mathrm{C}$ and stained with Annexin V-FITC conjugated with PI, then measured using flow cytometry. This assay was conducted according to the manufacturer's instructions (BD Biosciences).

Western blot analysis. Cells were collected at the time point and resuspended in $1 \%$ Triton X-100 supplemented with a 
Table I. Gene sequence of hammerhead ribozyme 138.

\begin{tabular}{llll}
\hline & \multicolumn{1}{c}{ 5' Binding arm } & Catalytic core & 3' Binding arm \\
\hline Rz138 sense & 5' CTAGATCCATTTAA & CTGATGAGTCCGTGAGGACGAA & ACATTCTTG 3' \\
Rz138 antisense & 5'GATCCAAGAATGT & TTCGTCCTCACGGACTCATCAG & TTAAATGGAT 3' \\
\hline
\end{tabular}

complete protease inhibitor cocktail (Boehringer Mannheim, Germany). Cell lysates were centrifuged at 13,000 rpm for $30 \mathrm{~min}$ at $4^{\circ} \mathrm{C}$, the supernatants were collected and stored at $-70^{\circ} \mathrm{C}$. Protein concentrations were determined using BCA protein assay kit (Pierce, Rockford).

Protein $(15 \mu \mathrm{g})$ of each sample in loading buffer was boiled for $4 \mathrm{~min}$, cooled in ice, then separated by sodium dodecyl sulfate polyacrylamide gel electrophoresis (SDSPAGE) using a $10 \%$ running gel with a $5 \%$ stacking gel at $100 \mathrm{~V}$ for $150 \mathrm{~min}$. Pre-stained molecular weight markers were used as standards. Proteins were transferred from the polyacrylamide gel to the nitrocellulose membrane by electro-blotting for $100 \mathrm{~min}$ in transfer buffer at a constant setting of $100 \mathrm{~V}$. Blots were blocked with 5\% non-fat dry milk in phosphate buffered saline, $\mathrm{pH} 7.6$, with $0.1 \%$ Tween20 buffer and then incubated with anti-caspase-12 antibody (Exalpha Biologicals, 1:1000)(16). Immunoreactivity was detected by the sequential incubation of horseradish peroxidase-conjugated secondary antibodies (Amersham Biosciences, 1:2000), and specific complexes were detected with the enhanced chemiluminescence (ECL) system (Amersham Life Science). Adjustment of protein loading onto gels was performed by re-probing with anti-ß-actin (Sigma; 1:5000). The density of bands was measured using Touching 2000 image analysis software.

Transfection of plasmids and induction of apoptosis in primary mouse hepatocytes. After isolation for 3 days, cells cultured in 6-well plates grew to $90 \%$ confluent before transfection. Each well was transfected with $4 \mu \mathrm{g}$ plasmid by Lipofectamine $^{\mathrm{TM}} 2000$ reagent (Invitrogen) using serum and antibiotic-free medium according to the manufacturer's instruction. Transfection efficiency was checked by observing green fluorescent protein (GFP) expressing cells under the fluorescence microscope. Cells transfected with pRz138, pEGFP-C1 and those untransfected were all incubated with $4 \mu \mathrm{mol} / 1 \mathrm{TG}$ to induce ER stress mediated apoptosis.

Ribozyme activity in primary mouse hepatocytes. To assess the functional activity of Rz138, semi-quantitative reverse transcription (RT)-PCR and Western blot analysis were used to demonstrate the ability of the ribozyme to down-regulate caspase-12 expression in cells. Total RNA of primary mouse hepatocytes transfected with pRz138, untransfected control and those transfected with pEGFP-C1 plasmid without ribozyme embedding, were obtained using a TRIzol kit (Promega). The $\beta$-actin was used as an internal control. The primers for caspase-12 were 5'-AGG ATG ATG GAC CTC AGA AG -3' (forward) and 5'-TCT CAG ACT CCG ACA GTT AG -3' (reverse). Primers for $\beta$-actin were 5'-CTA CAA

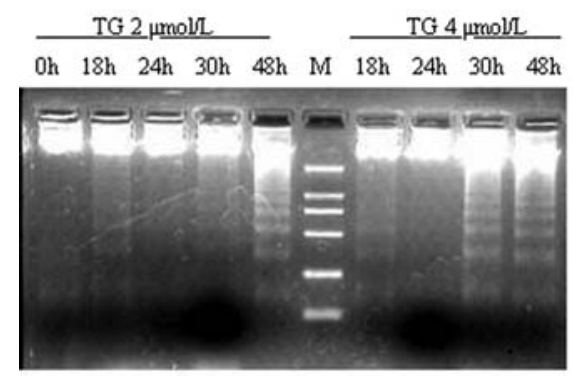

Figure 1. Detection of apoptosis by electrophoresis of fragmented DNA. DNA fragmentation can be detected when treated with $4 \mu \mathrm{mol} / \mathrm{l} \mathrm{TG}$ for $30 \mathrm{~h}$. When treated with $2 \mu \mathrm{mol} / 1 \mathrm{TG}$, it was detectable at $48 \mathrm{~h}$. Results are representative of three independent experiments.

TGA GCT GCG TGT GG-3' (forward) and 5'-CGG TCA GGA TCT TCA TGA GG-3' (reverse). The amplified DNA fragments were 587 bp (nt308-nt894) for caspase-12 mRNA, and $311 \mathrm{bp}$ (nt350-nt663) for $\beta$-actin mRNA. RT-PCR were carried out using $1.0 \mu \mathrm{g}$ total RNA as templates and a one step RT-PCR kit (Takara) for 32 PCR cycles according to the instruction, the annealing temperature was $56^{\circ} \mathrm{C}$. After electrophoresis on $1.6 \%$ agarose gels containing $0.5 \mu \mathrm{g} / \mathrm{ml}$ ethidium bromide, bands were analyzed by densitometry using Touching 2000 image analysis software. Procaspase-12 and $\beta$-actin proteins were obtained and analyzed as mentioned above.

Measurement of apoptosis in pRz138 transfected primary mouse hepatocytes. After plasmid transfection for $48 \mathrm{~h}$ and $30 \mathrm{~h}$ TG induction, floating and attached cells were stained with Hoechst 33258 in PBS $(2 \mu \mathrm{g} / \mathrm{ml})$ for $10 \mathrm{~min}$ at $37^{\circ} \mathrm{C}$, incubated in the dark. The nuclear morphology was observed using an Olympus fluorescence microscope. The number of apoptotic cells was calculated as a percentage of the total population.

\section{Results}

Apoptosis induction and caspase-12 processing. The optimal TG concentration for inducing cell apoptosis was determined by the detection of DNA fragmentation and flow cytometry analysis. Treatment with $4 \mu \mathrm{mol} / 1 \mathrm{TG}$ for $30 \mathrm{~h}$ was chosen as the concentration and time for inducing primary mouse hepatocytes apoptosis. Procaspase-12 processing during different incubation time was monitored using Western blot analysis.

In combination with the morphological changes, chromosomal DNA fragmentation is a hallmark of apoptotic 


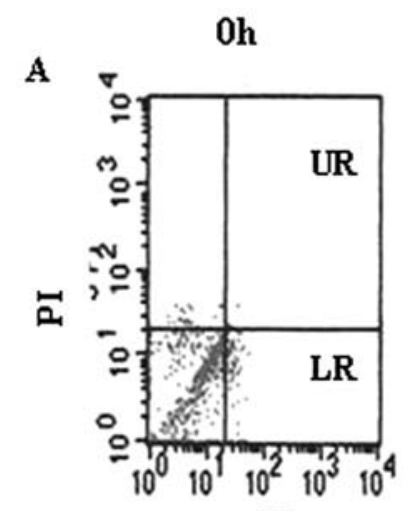

Annexin V FITC

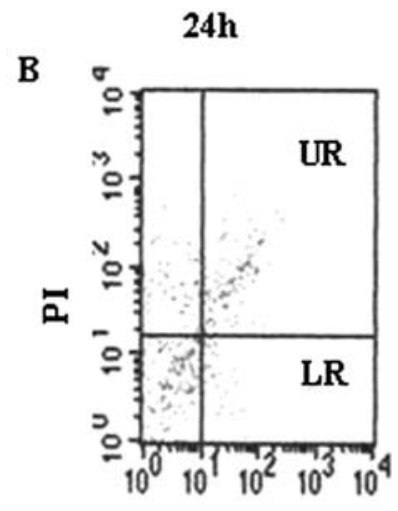

Annexin V FITC

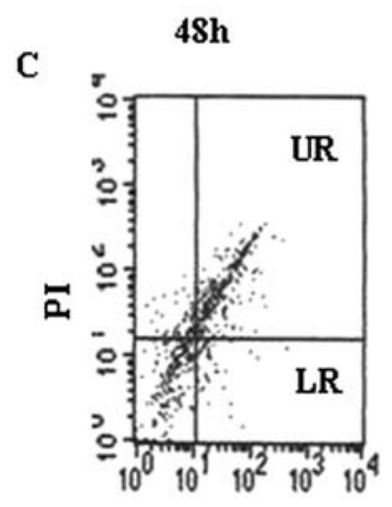

Annexin V FITC

Figure 2. Flow cytometry results of cell apoptosis. (A) Control untreated cells, $9.6 \%$ underwent apoptosis (UR+LR Annexin $\mathrm{V}^{+}, \mathrm{PI}^{+}$and $\mathrm{Annexin} \mathrm{V}^{+}$, $\left.\mathrm{PI}^{-}\right)$. (B) Induction with $4 \mu \mathrm{mol} / 1 \mathrm{TG}$ for $24 \mathrm{~h}, 41.2 \%$ cells underwent apoptosis (UR+LR). (C) Induction for $48 \mathrm{~h}, 51.4 \%$ cells were in late stage of apoptosis (UR Annexin $\left.\mathrm{V}^{+}, \mathrm{PI}^{+}\right)$. Data were obtained from three independent experiments.
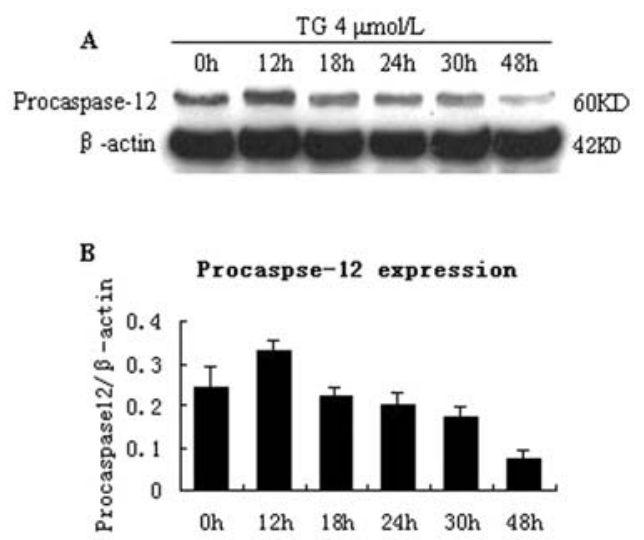

Figure 3. (A) Western blot analysis. TG treatment results in a time dependent processing of procaspase-12. (B) The graph shows results of densitometric quantification of the procaspase-12/ß-actin ratios. In $4 \mu \mathrm{mol} / 1$ TG treatment for $30 \mathrm{~h}$ procaspase-12 decreased $31.0 \%$ compared with the control. For $48 \mathrm{~h}$, the value was $70.3 \%$. The bars represent the standard deviation of three independent measurements.

cell death (37). As shown in Fig. 1, DNA fragmentation of primary mouse hepatocytes exposed to $4 \mu \mathrm{mol} / 1 \mathrm{TG}$ can be detected earlier than those exposed to $2 \mu \mathrm{mol} / 1 \mathrm{TG}$. The 'DNA ladder' was already obvious when treated with $4 \mu \mathrm{mol} / \mathrm{l}$ TG for $30 \mathrm{~h}$.

Cells treated with $4 \mu \mathrm{mol} / \mathrm{l} \mathrm{TG}$ at different time points were harvested and stained with fluorescein isothiocyanate (FITC)-labeled annexin $\mathrm{V}$ and propidium iodide (PI), then measured by flow cytometry. Annexin $\mathrm{V}$ binds to phosphatidylserine (PS) that is translocated during apoptosis from the inner to the outer leaflet of the cell membrane. Live cells with intact membranes are distinguished by their ability to exclude PI. Dual analysis was introduced using a quadrant dot blot, cells that stained negative for annexin V-FITC and PI were classified as live cells. 'Early apoptotic cells' were annexin V-FITC-positive only, and cells recognized as double-positive for annexin V-FITC and PI were considered late stage apoptosis or secondary necrosis $(38,39)$. The percentage of apoptotic cells increased in a time-dependent manner during TG induction. Without TG induction, 9.6\% cells underwent apoptosis, exposure to $4 \mu \mathrm{mol} / 1 \mathrm{TG}$ for $24 \mathrm{~h}$, $41.2 \%$ cells underwent apoptosis, $4 \mu \mathrm{mol} / 1 \mathrm{TG}$ incubation for $48 \mathrm{~h}, 51.4 \%$ cells were in late stage of apoptosis/secondary necrosis. It suggests that treatment with $4 \mu \mathrm{mol} / 1 \mathrm{TG}$ for $30 \mathrm{~h}$, $\sim 50 \%$ cells will undergo apoptosis. The results were consistent with those exhibited by DNA fragment detection (Fig. 2).

Treatment of cells with TG resulted in significant processing of procaspase-12. Western blot analysis showed at least a $70 \%$ reduction in the decline of procaspase- 12 in a time-dependent manner during $4 \mu \mathrm{mol} / 1 \mathrm{TG}$ treatment for $48 \mathrm{~h}$. These observations confirmed that procaspase-12 was activated through TG incubation and correlated with the appearance of morphological features of apoptosis (Fig. 3).

Transfection efficiency of $p R z 138$. Productive transfection was checked by observing green fluorescent protein (GFP) expressing cells under fluorescence microscope. The transfection efficiency was determined by calculating the GFP expressing cells in the whole population. The data showed that $47.7 \%$ of the cells were pRz138 positive after 3 days of transfection and $30 \mathrm{~h}$ incubation with $4 \mu \mathrm{mol} / 1 \mathrm{TG}$ (Fig. 4).

pRz138 inhibits the expression of caspase-12 in primary mouse hepatocytes. Hepatocytes transfected with pRz138, pEGFP$\mathrm{C} 1$ and those untransfected were all exposed to $4 \mu \mathrm{mol} / \mathrm{l} \mathrm{TG}$ for $30 \mathrm{~h}$ after transfection for 2 days. Total RNA extraction and RT-PCR were carried out as mentioned above, after electrophoresis and staining with ethidium bromide, the bands were quantified by densitometry. This showed that the level of caspase-12 mRNA in pRz138 transfected cells was decreased $37.8 \%$ compared with the untransfected control, while pEGFP-C1 have no obvious effect.

For Western blot analysis, the bands on the X-ray film were also quantified by densitometry. The $\beta$-actin was measured as an internal control. The results showed that the level of procaspase-12 protein was decreased $43.5 \%$ in pRz138 transfected cells compared with the untransfected control, pEGFP-C1 has no obvious effect.

In conclusion, transfection with pRz138 can significantly inhibit the expression of caspase-12 in primary mouse hepatocytes induced by TG compared with the untransfected 
A

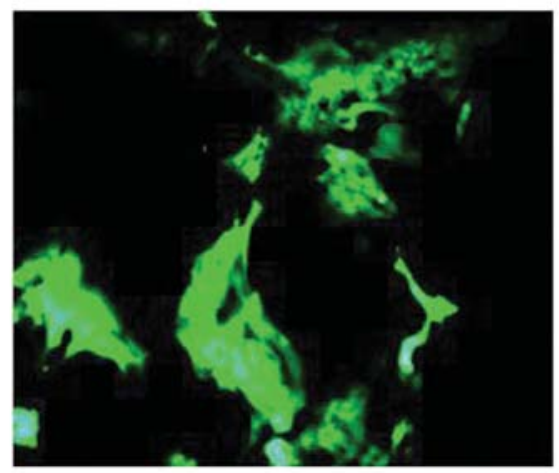

B

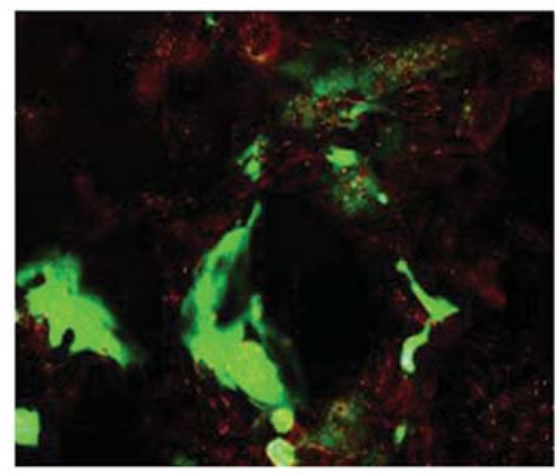

Figure 4. (A) Expression of GFP in pRz138 transfected primary mouse hepatocytes. (B) The same microscopic field view illuminated with fluorescent and white light. Cells were counted in 10 random fields of each sample. The data showed that $47.7 \%$ of the cells were pRz138 positive after 3 days of transfection (x100).

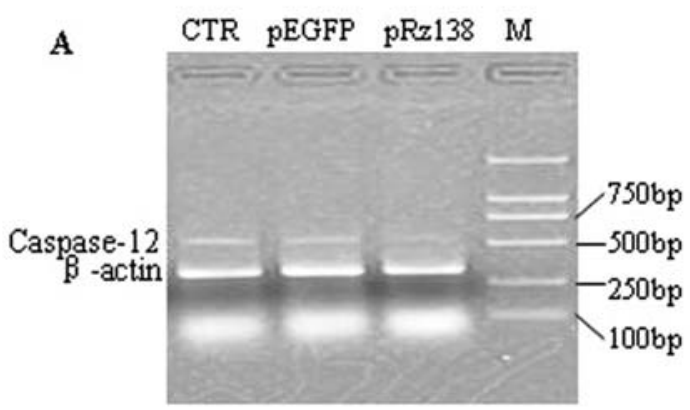

B

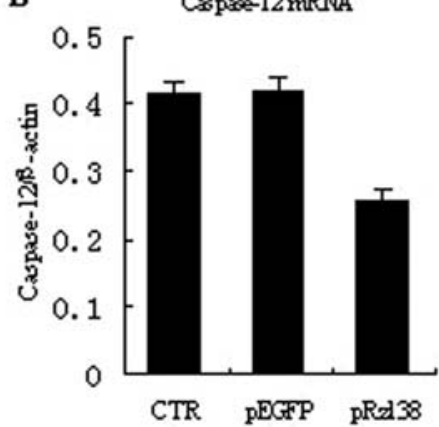

$\mathrm{C}$ CTR pEGFP pRz138

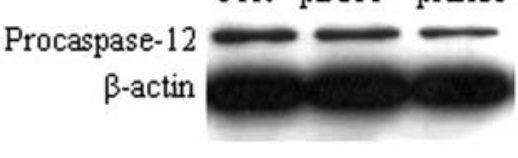

$\mathbf{D}$

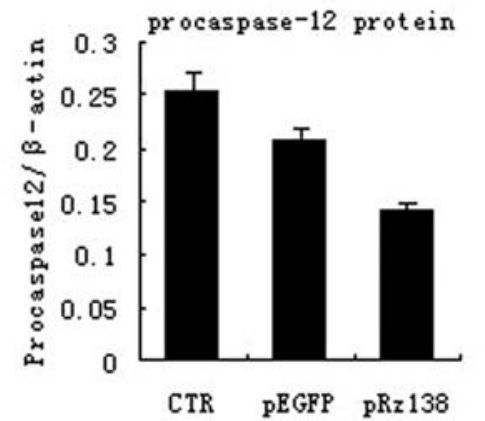

Figure 5. pRz138 decreased caspase-12 expression in TG treated hepatocytes. (A) RT-PCR results. Caspase-12 mRNA was decreased in pRz138 transfected cells. (B) The graph shows results of densitometric quantification of the caspase-12/ß-actin ratios. The level of caspase-12 mRNA was decreased $37.8 \%$ in pRz138 transfected cells. (C) Western blot analysis. The band of procaspase-12 protein decreased in pRz138 transfected cells. (D) Procaspase-12 protein in pRz138 transfected cells was decreased $43.5 \%$ compared with untransfected control (CTR), the values were normalized with the 3 -actin expression level, the bars represent the standard deviation of three independent measurements.

control and those transfected with pEGFP-C1 plasmid without Rz138 embedded (Fig. 5).

pRz138 reduces $T G$-induced primary mouse hepatocytes apoptosis. We chose Hoechst staining to evaluate the antiapoptotic effect of pRz138. Stained with Hoechst 33258 the healthy cell had an oval-shaped body, its chromatin occupied the majority of the cell body volume and it was stained dimly. The chromatin of an apoptotic cell was condensed and intensely stained. Cells were counted in 10 random fields of each sample. With primary mouse hepatocytes cultured for 6 days without TG induction and pRz138 transfection, the percentage of apoptotic cells was $12.7 \%$. With cells of the same cultured condition in additional $4 \mu \mathrm{mol} / 1 \mathrm{TG}$ induction for $30 \mathrm{~h}$, the amount of attached cells decreased significantly and $54.5 \%$ cells underwent apoptosis, while in cells transfected with pRz138, the percentage of apoptotic cells was $33.8 \%$ (Fig. 6). Transfection with pRz138 protected primary mouse hepatocytes from apoptosis induced by TG compared with TG treatment alone. Statistical significance was evaluated using paired Student's t-test $(\mathrm{P}<0.01)$.

\section{Discussion}

ER stress constitutes a physiological as well as pathophysiological stress stimulus, it can be provoked by a variety 
A

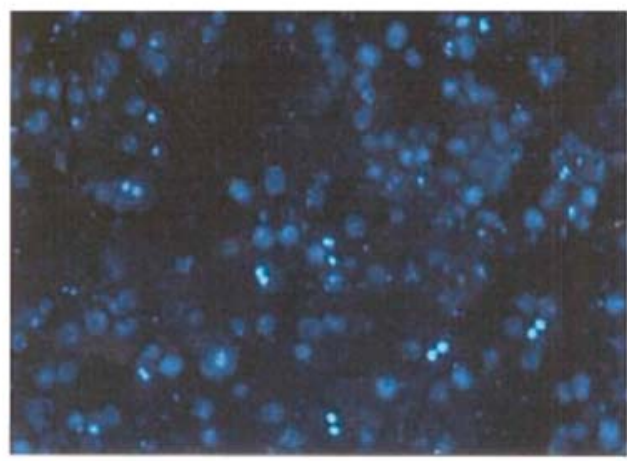

C

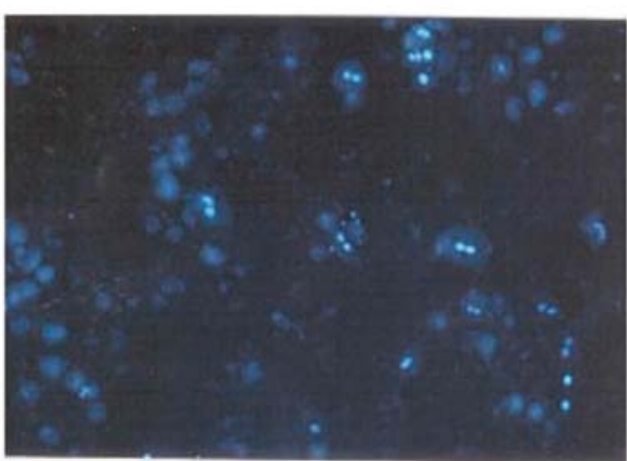

B

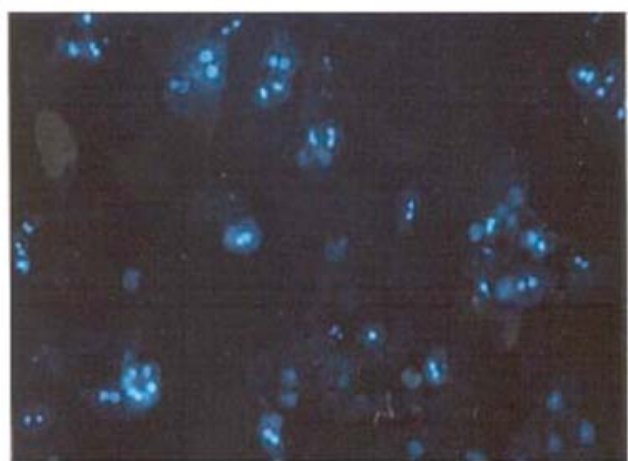

D

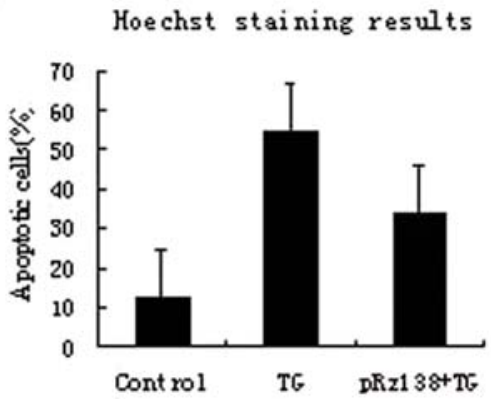

Figure 6. pRz138 inhibited TG induced apoptosis. Cells were stained with Hoechst 33258. (A) Without TG induction (control), 12.7\% cells underwent apoptosis (arrow). (B) TG induction alone (TG), 54.5\% cells underwent apoptosis. (C). Incubated with TG after pRz138 transfection (pRz138+TG), apoptotic cells was $33.8 \%$ (x200). (D) The graph shows results from three independent experiments.

of pathophysiological conditions, such as ischemia, viral infection, or mutations that impair protein folding and has been implicated as an initiator or contributing factor in numerous diseases, including Alzheimer's disease, Parkinson's disease, and type 2 diabetes, ER stress also plays an important role in liver cirrhosis and many other liver diseases $(40,41)$. The ER stress response can promote cellular repair and sustain survival by reducing the load of unfolded proteins through global attenuation of protein synthesis and/or up-regulation of chaperones, enzymes, and structural components of the ER (42). When ER stress is overwhelming, cells undergo apoptosis. This was initially reported to be mediated by caspase- 12 which primarily associated with the ER membranes (2). Caspase-12 has been shown to be activated by cytotoxic agents, ischemic insult, viral infection and in models of neurodegenerative diseases $(24,43,44)$.

Activation of caspases is a central mechanism in the apoptotic cell death process. The caspase family involved in apoptosis is broadly divided into two groups: initiator caspases (caspase-8,-9,-12) and effector caspases (caspase-3,-6,-7). Initiator caspases undergo auto-processing for activation in response to apoptotic stimuli. Active initiator caspases in turn process precursors of the effector caspases responsible for dismantling cellular structures and proteins (45). Activation of caspase-12 from procaspase- 12 is specifically induced by insult to the endoplasmic reticulum. Caspase-12 is ubiquitously expressed in mouse tissue, it is expressed at high levels in the muscle, liver and kidney, and at a moderate level in the brain (2). It is also expressed in many human cell lines. Active caspase-12 cleaves and activates procaspase-9, the activated caspase- 9 catalyzes procaspase- 3 , then ER stress triggers a specific apoptotic cascade involving caspase- 12, 9 and 3 $(2,46)$. Caspases are central to normal programmed cell death and injury-dependent apoptosis, so any therapy that manipulates caspase activity must take into account the possible effects on tissue homeostasis. In this regard, caspase-12 appears to have a strong advantage as a target over other caspases. In contrast to other caspase knockout, caspase-12 deficient mice have no noticeable developmental or behavioral defects, and have a normal incidence of tumors. Thus, caspase-12 is probably not essential in normal developmental death or tumorigenesis $(46,47)$. Combined with that caspase-12-/- cells are more resistant to ER stressinduced apoptotic signals than caspase-12+/- cells, and caspase-12 null mice are resistant to the toxic effects of ER stress $(2,46)$. It will be a promising potential target for inhibition of ER stress mediated cell apoptosis with few side effects.

Today, many nucleic acid enzymes are used in gene therapy and gene regulation. The trans-acting ribozymes recognize their RNA substrates via formation of WatsonCrick base pairs, and they cleave these RNAs in a sequencespecific manner. The activity of hammerhead ribozyme was significantly higher than that of all the other ribozymes in vitro (48). In the present study, we designed and synthesized hammerhead ribozymes against caspase- 12 mRNA under the control of a U6 snRNA promoter. The U6 SnRNA Pol III transcripts were normally retained in the nucleus and expressed at high levels, transcripts were protected at their 3'-ends and Rev-binding RNAs and had certain advantages (49). It is a strong promoter for the expression of small RNAs in vivo. Transcription by RNA polymerase III is easily stopped by a 
string of Us, leaving the transcripts uncapped and untailed and the transcribed ribozyme RNAs do not interfere with productive annealing to the target (23).

The transfection efficiency was a crucial matter for delivery of gene therapy. Watanabe et al (50) have reported that cation liposomes induced highly efficient transfection into primary cultured mouse hepatocytes compared with DEAE-dextran, calcium phosphate and cation multilamellar liposomes, the highest transfection rate achieved more than $60 \%$ of the total cells and remained at a high level from 6 to $48 \mathrm{~h}$ after the start of incubation. Our study attained an average $47.7 \%$ of transfection efficiency. However, for a better understanding of pRz138's function in cultured cells, the transfection efficiency still needs to be improved.

The RT-PCR and Western blot results showed that as a specific inhibitor of caspase-12, Rz138 can down-regulate the expression of caspase-12 in primary mouse hepatocytes.

We assessed and quantified the anti-apoptosis effect of pRz138 by Hoechst 33258 assay. Staining with Hoechst 33258 has been used to identify specific apoptotic features, and it was reported that the staining pattern observed with this method was similar to that produced by TUNEL, indicating that both techniques may provide a similar estimate of apoptosis (51). Our results showed that Rz138 had significant activity in cultured cells and it can partly protect primary mouse hepatocytes from apoptosis induced by TG. Cell viability as evaluated by MTT assay also indicated that transfection with pRz138 increased the number of viable cells in TG induced cells compared with TG treatment alone (data not shown). We did not expect $100 \%$ inhibition in our transient expression assays because a) not all the cells were transfected by the plasmids, b) many protein factors are involved in living cells which will disturb the ribozyme to cleave target mRNAs, c) ribozyme and substrate will not stay in the same subcellular location as expected, and d) other apoptotic pathways may be involved in TG-induced apoptosis.

In conclusion, TG induced the degradation of caspase-12 and cell apoptosis; Rz138 can down-regulate caspase-12 expression in primary mouse hepatocytes and can protect the cells from ER stress induced apoptosis. These results suggest that Rz138 has the potential to be a promising caspase-12 inhibitor in treating diseases associated with excessive ER stress.

It was reported recently that the deletion of caspase-12 renders animals resistant to sepsis and enhances bacterial clearance (52). It will be very useful to test whether Rz138 also has potential in treating severe bacterial infection through cleavage of caspase- 12 mRNA.

\section{Acknowledgements}

This project is supported by the National Natural Science Foundation (Nos. 30170850 and 30371268).

\section{References}

1. Thompson CB: Apoptosis in the pathogenesis and treatment of disease. Science 267: 1456-1462, 1995.

2. Nakagawa T, Zhu H and Morishma N: Caspase-12 mediates ER-specific apoptosis and cytotoxicity by amyloid- $\beta$. Nature 403: 98-103, 2000.
3. Masud A, Mohapatra A, Lakhani SA, Ferrandino A, Hakem R and Flavell RA: Endoplasmic reticulum stress-induced death of mouse embryonic fibroblasts requires the intrinsic pathway of apoptosis. J Biol Chem 282: 14132-14139, 2007.

4. Luthra S, Dong J, Gramajo AL, Chwa M, Kim DW, Neekhra A, Kuppermann BD and Kenney MC: 7-Ketocholesterol activates caspases-3/7, -8 , and -12 in human microvascular endothelial cells in vitro. Microvasc Res 75: 343-350, 2008.

5. Crosby KM, Connell BJ and Saleh TM: Estrogen limits ischemic cell death by modulating caspase-12-mediated apoptotic pathways following middle cerebral artery occlusion. Neuroscience 146: 1524-1535, 2007.

6. Manekeller S, Schuppius A, Stegemann J, Hirner A and Minor T: Role of perfusion medium, oxygen and rheology for endoplasmic reticulum stress-induced cell death after hypothermic machine preservation of the liver. Transpl Int 21: 169-177, 2008.

7. Ji C and Kaplowitz N: Betaine decreases hyperhomocysteinemia, endoplasmic reticulum stress, and liver injury in alcohol-fed mice. Gastroenterology 124: 1488-1499, 2003.

8. Werstuck GH, Lentz SR, Dayal S, et al: Homocysteine-induced endoplasmic reticulum stress causes dysregulation of the cholesterol and triglyceride biosynthetic pathways. J Clin Invest 107: 1263-1273, 2001.

9. Xu Z, Jensen G and Yen TS: Activation of hepatitis B virus S promoter by the viral large surface protein via induction of stress in the endoplasmic reticulum. J Virol 71: 7387-7392, 1997.

10. Foo NC, Ahn BY, Ma X, Hyun W and Yen TS: Celluler vacuolization and apoptosis induced by HBV large surface protein. Hepatology 36: 1400-1407, 2002.

11. Rodrigues CM, Fan G, Ma X, Kren BT and Steer CJ: A novel role for ursodexycholic acid in inhibiting apoptosis by modulating mitochondrial membrane perturbation. J Clin Invest 101: 2790-2799, 1998.

12. Pavio N, Romano PR, Graczyk TM, Feinstone SM and Taylor DR: Protein synthesis and endoplasmic reticulum stress can be modulated by the hepatitis $C$ virus envelope protein $E 2$ through the eukaryotic initiation factor 2alpha kinase PERK. J Virol 77: 3578-3585, 2003.

13. Tardif KD, Mori K and Siddiqui A: Hepatitis $C$ virus subgenomic replicons induce endoplasmic reticulum stress activating an intracellular signaling pathway. J Virol 76: 7453-7459, 2002.

14. Liu H, Miller E, van de Water B and Stevens JL: Endoplasmic reticulum stress proteins block oxidant-induced $\mathrm{Ca}^{2+}$ increases and cell death. J Biol Chem 273: 12858-12862, 1998.

15. Paschen W: Dependence of vital cell function on endoplasmic reticulum calcium levels: implications for the mechanisms underlying neuronal cell injury in different pathological states. Cell Calcium 29: 1-11, 2001.

16. Xie Q, Khaoustov VI, Chung CC, et al: Effect of tauroursodeoxycholic acid on Endoplasmic Reticulum stress-induced caspase-12 activation. Hepatology 36: 592-601, 2002.

17. Wertz IE and Dixit VM: Characteriztion of calcium releaseactivated apoptosis of LNCaP prostate cancer cells. J Biol Chem 275: 11470-11477, 2000.

18. Liu H, Bowes RC, Van de Water B, Sillence C, Nagelkerke JF, Stevens JL: Endoplasmic reticulum chaperones GRP78 and calreticulin prevent oxidative stress, $\mathrm{Ca}^{2+}$ disturbances, and cell death in renal epithelial cells. J Biol Chem 272: 21751-21759, 1997.

19. Voccoli V, Mazzoni F, Garcia-Gil M and Colombaioni L: Serum-withdrawal-dependent apoptosis of hippocampal neuroblasts involves $\mathrm{Ca}^{++}$release by endoplasmic reticulum and caspase-12 activation. Brain Res 1147: 1-11, 2007.

20. Cryns V and Yuan J: Proteases to die for. Genes Dev 12: 1551$1570,1998$.

21. Slee EA, Adrain C and Martin SJ: Serial killer: ordering caspase activation events in apoptosis. Cell Death Differ 6: 1067-1074, 1999.

22. Bitko V and Barik S: An endoplasmic reticulum-specific stressactivated caspase (caspase-12) is implicated in the apoptosis of A549 epithelial cells by respiratory syncytial virus. J Cell Biochem 80: 441-454, 2001.

23. Xu R-H, Liu J, Chen X-W, et al: Ribozyme-mediated inhibition of Caspase-3 activity reduces apoptosis induced by 6 hydroxydopamine in PC12 cells. Brain Res 899: 10-19, 2001.

24. Larner SF, Hayes RL, McKinsey DM, Pike BR and Wang KK: Increased expression and processing of caspase-12 after traumatic brain injury in rats. J Neurochem 88: 78-90, 2004. 
25. Lai D, Fu L, Weng S, Qi L, Yu C, Yu T, Wang H and Chen W: Blocking caspase-3 activity with a U6 SnRNA promoter-driven ribozyme enhances survivability of $\mathrm{CHO}$ cells cultured in low serum medium and production of interferon-beta. Biotechnol Bioeng 85: 20-28, 2004.

26. Verma S, Vaish NK and Eckstein F: Structure-function studies of the hammerhead ribozyme. Curr Opin Chem Biol 1: 532-536, 1997.

27. Wong-Staal F, Poeschla EM and Looney DJ: A controlled, Phase 1 clinical trial to evaluate the safety and effects in HIV-1 infected humans of autologous lymphocytes transduced with a ribozyme that cleaves HIV-1 RNA. Hum Gene Ther 9: 2407-2425, 1998.

28. Suyama E, Kawasaki H and Taira K: Characterization of proapoptotic gene Bak using hammerhead ribozymes. Nucleic Acids Res (Suppl 1): 207-208, 2001.

29. Jiang S, Xie Q, Zhang W, et al: Preparation of anti-mouse caspase-12 mRNA hammerhead riboyme and identification of its activity in vitro. World J Gastroenterol 11: 4094-4097, 2005.

30. Klaunig JE, Goldblatt PJ, Hinton DE, Lipsky MM, Chacko J and Trump BF: Mouse liver cell culture I: hepatocyte isolation. In Vitro 17: 913-925, 1981.

31. Bassi GS, Mollegaard NE, Murchie AI and Lilley DM: RNA folding and misfolding of the hammerhead ribozyme. Biochemistry 11: 3345-3354, 1999.

32. Lin JS, Song YH, Kong XJ, Li B, Liu NZ, Wu XL and Jin YX: Preparation and identification of anti-transforming growth factor B1 U1 small nuclear RNA chimeric ribozyme in vitro. World J Gastroenterol 9: 572-577, 2003.

33. Mendoza-Maldonado R, Zentilin L, Fanin R and Giacca M: Purging of chronic myelogenous leukemia cells by retrovially expressed anti-bcr-abl ribozymes with specific cellular compartmentalization. Cancer Gene Ther 9: 71-86, 2002.

34. Pennati M and Colella G: Ribozyme-mediated attenuation of surviving expression sensitizes human melanoma cells to cisplatin-induced apoptosis. J Clin Invest 109: 285-286, 2002.

35. Sriram B and Thakral D: Target cleavage of hepatitis E virus 3' end RNA mediated by hammerhead ribozymes inhibits viral RNA replication. Virology 312: 350-358, 2003.

36. Weinberg $M$, Passman $M$, Kew $M$ and Arbuthnot P: Hammerhead ribozyme-mediated inhibition of hepatitis $B$ virus $\mathrm{X}$ gene expression in cultured cells. J Hepatol 33: 142-151, 2000 .

37. Matassov D, Kagan T, Leblanc J, Sikorska M and Zakeri Z: Measurement of apoptosis by DNA fragmentation. Methods Mol Biol 282: 1-17, 2004.

38. Wolbers F, Buijtenhuijs P, Haanen $\mathrm{C}$ and Vermes I: Apoptotic cell death kinetics in vitro depend on the cell types and the inducers used. Apoptosis 9: 385-392, 2004.
39. Wising C, Azem J, Zetterberg M, Svensson LA, Ahlman K and Lagergard $\mathrm{T}$ : Induction of apoptosis/necrosis in various human cell lineages by Haemophilus ducreyi cytolethal distending toxin. Toxicon 45: 767-776, 2005.

40. Rao RV, Castro-Obregon S and Frankowski H: Coupling endoplasmic reticulum stress to the cell death program. J Biol Chem 277: 21836-21842, 2002.

41. Rutishauser J and Spiess M: Endoplasmic reticulum storage diseases. Swiss Med Wkly 132: 211-222, 2002.

42. Kaufman R J: Orchestrating the unfolded protein response in health and disease. J Clin Invest 110: 1389-1398, 2002.

43. Lamkanfi M, Kalai $M$ and Vandenabeele P: Caspase-12: an overview, Cell Death Differ 11: 365-368, 2004

44. Terai K, Hiramoto Y, Masaki M, Sugiyama S, Kuroda T, Hori M, Kawase I and Hirota H: AMP-activated protein kinase protects cardiomyocytes against hypoxic injury through attenuation of endoplasmic reticulum stress. Mol Cell Biol 25: 9554-9575, 2005.

45. Morishima N, Nakanishi K and Takenouchi H: An endoplasmic reticulum stress-specific caspase cascade in apoptosis. J Biol Chem 277: 34287-34294, 2002.

46. Mehmet H: Caspase find a new place to hide. Nature 403: 2930, 2000.

47. Zheng TS, Hunot S, Kuida K and Flavell RA: Caspase knockouts: matters of life and death. Cell Death Differ 6: 10431053, 1999.

48. Kato Y, Kuwabara T and Warashima M: Relationships between the activities in vitro and in vivo of various kinds of ribozyme and their intracellular localization in mammalian cells. J Biol Chem 276: 15378-15385, 2001.

49. Vaish NK, Kore AR and Eckstein F: Recent developments in the hammerhead ribozyme field. Nucleic Acids Res 26: 5237-5242, 1998 .

50. Watanabe Y, Nomoto H, Takezawa R and Miyoshi N, Akaike T: Highly efficient transfection into primary cultured mouse hepatocytes by use of cation-liposomes: an application for immunization. J Biochem 116: 1220-1226, 1994.

51. Eldadah BA, Ren RF and Faden AI: Ribozyme-mediated inhibition of caspase-3 protects cerebellar granule cells from apoptosis induced by serum-potassium deprivation. J Neurosci 20: 179-186, 2000.

52. Saleh M, Mathison JC, Wolinski MK, Bensinger SJ, Fitzgerald P, Droin N, Ulevitch RJ, Green DR and Nicholson DW: Enhanced bacterial clearance and sepsis resistance in caspase-12-deficient mice. Nature 440: 1064-1068, 2006. 